\title{
'MEDICAL EDUCATION TOURISM' IN NEPAL
}

The first medical college, the Institute of Medicine, in Nepal was established at Kathmandu. The medical course at the Institute of Medicine was started in $1978 .{ }^{1}$ This institute was supported by the Government of Japan for establishment of a Teaching Hospital. The first batch of Institute of Medicine's basic community oriented doctor came out in 1984. ${ }^{2}$ Then the B. P. Koirala Institute of Health Sciences (BPKIHS) was established in 1993 at Dharan with the help of the Government of India.

After 1993, the idea was mooted that having private medical colleges will not only bring about a boost in the economy but that a good service sector could be created in the health field. ${ }^{1}$ Besides decreasing the amount of money being spent outside of the country for the health care of the Nepalese, the government would be providing health services to a substantial portion of the population, at no cost to itself. Further this would not only save the money spent by Nepalese students in getting the education outside the country but also earn the foreign exchange by getting admission of the foreign students in the medical institutes in the country. Since then, many private medical colleges were established in the country, like Manipal College of Medical Sciences (MCOMS), Pokhara in 1994, College of Medical Sciences - Nepal, Bharatpur, in 1996, ${ }^{3}$ Nepalgunj Medical College, Nepalgunj in 1997, Kathmandu Medical College, Kathmandu in 1997, Nepal Medical College, Kathmandu in 1997. Similarly, Universal College of Medical Sciences, Bhairahawa and People's Dental College, Kathmandu were started in 1999 and National Medical College, Birgunj in 2001, Kathmandu University Medical School (KUMS) in 2001 and Janaki Medical College in 2003. Besides these, there are plans for starting medical colleges in different parts of the country. Some of the towns are expecting more than one medical college.

Out of these, Institute of Medicine and BP Koirala Institute of Health Sciences are public medical colleges, rest are private ones. Not surprisingly these medical colleges admit substantially foreign students from different countries. The students are from different countries like India, Maldives, Sri Lanka, Gulf countries, Japan etc. Majority of students in most of the private medical colleges are foreigners. Thus, such foreign remittance has become a good source of earning for the country. This is obviously a matter of importance for planners and policy makers and the government. If the medical colleges are not recognized by the Medical Councils of their respective countries, students may stop to come and this will be a great loss to the country. The Government, Universities and Medical Council have to see that the quality of the medical colleges is maintained.

Nepal Medical Council has laid down guidelines to maintain standard requirements for medical colleges. ${ }^{1,4}$ Nepal Medical Council has laid down the ratio of hospitals beds per students, including departments necessary with beds in different specialties. ${ }^{5}$ As per the guidelines there should be a teacher of Professor, Associate Professor, or Lecturer level for every 15 students in major departments. ${ }^{1}$ The faculty position in any one institute is usually recognized by the other institute in the country, so the requirements for the faculty position should be of the standard. The Nepal Medical Council has similarly laid down the standard requirements in regards to accommodation, staff, equipment required etc. ${ }^{4}$ Nepal Medical Council has been, thus, working on the Accreditation Document for the Medical and Dental Education in Nepal. The requirement of Medical Education Unit in each medical institute is also being emphasized. Thus, the quality of curriculum, basic training requirements etc have to be maintained uniformly in the country.

In summary, it is important for everyone to understand the value of medical institutes in the country as 'Medical Education Tourism' is not only saving but also earning the substantial amount of foreign exchange. It, like General Tourism, is obviously important for the economy of the country. In regards to exporting carpet and other materials from the country, people, planners and politicians understand the importance of maintenance of the quality of products. Let everyone do similarly understand the importance of maintenance of the quality of "Medical Education Tourism' of the country!

\section{REFERENCES}

1. Dixit H. The Quest for Health. Second Edition. Kathmandu: Educational Enterprise (P) Ltd. 1999: 177-89.

2. Innovative Programmes of Medical Education in South-East Asia 1993, WHO, SEARO Publication No. 21.

3. SHRUTI-97 Souvenir, College of Medical Science-Nepal, Bharatpur, 1997.

4. Nepal Medical Council. Minimum Standard Requirements for Starting a Medical School for 100 Admissions Annually. Kathmandu: Nepal Medical Council, 1995.

5. Nepal Medical Council. Nepal Medical Council Guidelines. Kathmandu: Nepal Medical Council, 1997.

出 20 


\title{
ANTIMICROBIAL UTILIZATION PATTERN IN A TEACHING DISTRICT HOSPITAL OF NEPAL
}

\author{
Das B P*, Sethi A*, Rauniar G P
}

\section{ABSTRACT}

The objective of this study was to monitor the antimicrobial utilization in a teaching district hospital and to compare it against the rational antimicrobial use guidelines.

Total 106 data sheet of indoor patients admitted in year 2001 were randomly chosen and analyzed. The number of antimicrobials prescribed in every prescription was taken into account to calculate the incidence of use of more than one antimicrobial agent. The Patient sheet included patient information, drug information (name, indication, dose, frequency and duration of antimicrobial use), diagnosis or provisional diagnosis and investigations. Prescription auditing was done on the selected prescriptions.

Male: female ratio was 40:65. In 90.57\% diagnosis was established empirically and in only $4.7 \%$ patients confirmatory diagnosis was made using relevant investigations. The antimicrobials were prescribed chiefly for respiratory tract infections (RTI -26.41\%), gastrointestinal infections (GIT -22.64\%), genital tract infection (13.20\%), wound infections $(\mathbf{8 . 4 8 \%})$, urinary tract infections (UTI-6.6\%), bone $\&$ joint infections $(4.71 \%)$ and miscellaneous (Typhoid, Malaria, meningitis etc-17.92\%). The causative organism was confirmed using culture $\&$ sensitivity tests in only one patient $(0.94 \%)$ out of 106 patients. In $77(69.8 \%)$ patient prescriptions suspected pathogenic organisms were mentioned. These were streptococcus (29.58\%), Chlamydia (11.27\%), mycoplasma (18.30\%), staphylococcus $(23.94 \%)$, E. coli $(32.39 \%)$ and S. typhi (15.49\%). In 35 prescriptions (33.01) no causative organism was mentioned. It was further observed that a total 452 drugs (i.e. 4.26 drugs per patient), out of this only 165 antimicrobials (1.55 antimicrobials per patient) were prescribed in the 106 enrolled prescriptions. On further analysis of antimicrobials use, it was observed that most patients were prescribed a single antimicrobial agent (58.49\%), two AMAs (24.52\%), three AMAs (13.20\%) and least was four AMAs (3.77\%).The antimicrobials were given prophylactically in $14(\mathbf{1 3 . 1 0 \%})$ and therapeutically in $85(\mathbf{8 0 . 1 8 \%})$ prescriptions. The use of antimicrobials were not indicated in $\mathbf{7}(6.60 \%)$ prescriptions.. In 91 cases $(\mathbf{8 5 . 8 5 \%})$ duration for which AMAs were considered was not mentioned. Ampicillin + cloxacillin $(12.12 \%)$, ciprofloxacin $(10.30 \%)$, metronidazole $(10.30 \%)$, amoxycillin (10.30\%) and cephalosporins $(\mathbf{2 5 . 4 7 \%})$ were the most frequently prescribed AMAs. A fixed dose combination of ampicillin \& cloxacillin, ciprofloxacin and metronidazole were recurrently prescribed while gentamicin, cotrimoxazole and crystalline penicillin were relatively least prescribed antimicrobials agents.

Inappropriate and irrationality in the Antimicrobial prescribing pattern was observed.

Key Words: Antimicrobial agent, Respiratory tract infection (RTI), urinary tract infection (UTI), gastrointestinal infection, prophylaxis, therapeutic.

${ }^{*}$ B.P. Koirala Institute of Health Sciences, Dharan, Nepal.

Address for correspondence : Dr. B. P. Das

B.P. Koirala Institute of Health Sciences, Dharan, Nepal.

Email: bpdas2000@yahoo.com 